\title{
Common Fixed Points of Generalized Cocyclic Mappings in Complex Valued Metric Spaces
}

\author{
Mujahid Abbas, ${ }^{1,2}$ Manuel De la Sen, ${ }^{3}$ and Talat Nazir ${ }^{4}$ \\ ${ }^{1}$ Department of Mathematics, King Abdulaziz University, P.O. Box 80203, Jeddah 21589, Saudi Arabia \\ ${ }^{2}$ Department of Mathematics and Applied Mathematics, University of Pretoria, Lynnwood Road, Pretoria 0002, South Africa \\ ${ }^{3}$ Institute of Research and Development of Process (IIDP), Faculty of Science and Technology, University of the Basque Country, \\ 48940 Leioa, Spain \\ ${ }^{4}$ Department of Mathematics, COMSATS Institute of Information Technology, Abbottabad 22060, Pakistan
}

Correspondence should be addressed to Manuel De la Sen; manuel.delasen@ehu.es

Received 19 August 2015; Accepted 18 October 2015

Academic Editor: Victor S. Kozyakin

Copyright (C) 2015 Mujahid Abbas et al. This is an open access article distributed under the Creative Commons Attribution License, which permits unrestricted use, distribution, and reproduction in any medium, provided the original work is properly cited.

We present fixed point results of mappings satisfying generalized contractive conditions in complex valued metric spaces. As an application, we obtain a common fixed point of a pair of weakly compatible mappings. Some common fixed point results of generalized contractive-type mappings involved in cocyclic representation of a nonempty subset of a complex valued metric space are also obtained. Some examples are also presented to support the results proved herein. These results extend and generalize many results in the existing literature.

\section{Introduction and Preliminaries}

Fixed point theory is one of the famous and traditional theories in mathematics that provide important and traditional tools for proving the existence of solutions of many problems in both applied and pure mathematics. In metric fixed point theory, the interplay between contractive condition and the existence and uniqueness of a fixed point has been very strong and fruitful. There are many applications to the study of fixed points of mappings which satisfy contractive conditions related to the solutions of differential and integral equations [1-3]. Fixed point results for mappings inspired and motivated the development of many other important kinds of points like equilibrium points, coincidence points, periodic points, sectional points, intersection points, and so forth.

Banach's fixed point theorem is one of the basic and most widely applied fixed point theorems in mathematical analysis. Kirk et al. [4] established fixed point theorems for mappings satisfying cyclical contractive conditions. The cyclic contraction mappings need not be continuous, while the Banach contraction mappings are continuous mappings. Păcurar and Rus [5] proved some fixed point results for cyclic weakly contractive mappings. Karapinar [6] obtained fixed point results for cyclic weakly contractive mappings. Derafshpour and Rezapour [7] proved some results on the existence of best proximity points of cyclic contractions. Recently, Abbas et al. [8] established some common fixed point results of rational-type cocyclic mappings in the setup of multiplicative metric spaces. For further interesting results in this direction, we refer readers to [9-17]. There are many generalizations of metric spaces such as partial metric spaces, generalized metric spaces, cone metric spaces, and quasimetric spaces. Recently, Azam et al. [18] obtained the generalization of Banach's contraction principal by introducing the concept of complex valued metric space and established some common fixed point theorems for mappings involving rational expressions which are not meaningful in cone metric spaces. Later, some other useful results appeared in [19-24]. Abbas et al. [25] obtained the well-posedness and periodic point property of mappings satisfying a rational inequality in an ordered complex valued metric space. Recently, Nashine et al. [26] proved common fixed point theorems under rational contractions in complex valued metric spaces.

In this paper, we establish fixed point result of a generalized contractive mapping in the setup of complex valued metric spaces. Further, by employing this result, a common fixed 
point of a pair of weakly compatible mappings is obtained. We study the sufficient conditions for the existence of common fixed points of pair of rational contractive-type mappings involved in cocyclic representation of a nonempty subset of a complex valued metric space. Our results generalize and extend comparable results.

By $\mathbb{R}, \mathbb{R}^{+}, \mathbb{C}, \mathbb{C}^{+}$, and $\mathbb{N}$ we denote the set of all real numbers, the set of all nonnegative real numbers, the set of all complex numbers, the set of all nonnegative complex numbers, and the set of all natural numbers, respectively.

Consistent with Azam et al. [18], the following definitions and results will be needed in the sequel.

Let $z_{1}, z_{2} \in \mathbb{C}$. Define a partial order $\preceq$ on $\mathbb{C}$ as follows:

$z_{1} \preceq z_{2}$ if and only if $\operatorname{Re}\left(z_{1}\right) \leq \operatorname{Re}\left(z_{2}\right), \operatorname{Im}\left(z_{1}\right) \leq \operatorname{Im}\left(z_{2}\right)$.

It follows that $z_{1} \preceq z_{2}$ if one of the following conditions is satisfied:

(1) $\operatorname{Re}\left(z_{1}\right)=\operatorname{Re}\left(z_{2}\right), \operatorname{Im}\left(z_{1}\right)<\operatorname{Im}\left(z_{2}\right)$.

(2) $\operatorname{Re}\left(z_{1}\right)<\operatorname{Re}\left(z_{2}\right), \operatorname{Im}\left(z_{1}\right)=\operatorname{Im}\left(z_{2}\right)$.

(3) $\operatorname{Re}\left(z_{1}\right)<\operatorname{Re}\left(z_{2}\right), \operatorname{Im}\left(z_{1}\right)<\operatorname{Im}\left(z_{2}\right)$.

(4) $\operatorname{Re}\left(z_{1}\right)=\operatorname{Re}\left(z_{2}\right), \operatorname{Im}\left(z_{1}\right)=\operatorname{Im}\left(z_{2}\right)$.

In particular, we will write $z_{1} \preceq z_{2}$ if one of (1), (2), and (3) is satisfied and we will write $z_{1} \prec z_{2}$ if only (3) is satisfied.

The partial order $\preceq$ on $\mathbb{C}$ satisfies the following properties:

(i) If $\theta \preceq z_{1} \preceq z_{2}$, then $\left|z_{1}\right| \leq\left|z_{2}\right|$, where $\theta$ is the zero of complex number.

(ii) $z_{1} \preceq z_{2}$ is equivalent to $z_{1}-z_{2} \preceq \theta$.

(iii) If $z_{1} \preceq z_{2}$ and $r \geq 0$ is a real number, then $r z_{1} \preceq r z_{2}$.

(iv) If $\theta \preceq z_{1}$ and $\theta \preceq z_{2}$ with $z_{1}+z_{2} \neq \theta$, then $z_{1}^{2} /\left(z_{1}+\right.$ $\left.z_{2}\right) \preceq z_{1}$.

(v) $\theta \preceq z_{1}$ and $\theta \preceq z_{2}$ do not imply $\theta \preceq z_{1} z_{2}$.

(vi) $\theta \preceq z_{1}$ does not imply $\theta \preceq 1 / z_{1}$. Moreover, if $\theta \preceq z_{1}$ and $\theta \preceq 1 / z_{1}$, then $\operatorname{Im}\left(z_{1}\right)=0$.

Definition 1. Let $X$ be a nonempty set. Suppose that the mapping $d: X \times X \rightarrow \mathbb{C}$ satisfies the conditions

(1) $\theta \preceq d(x, y)$ for all $x, y \in X$ and $d(x, y)=\theta$ if and only if $x=y$;

(2) $d(x, y)=d(y, x)$ for all $x, y \in X$;

(3) $d(x, y) \preceq d(x, z)+d(z, y)$ for all $x, y, z \in X$.

Then $d$ is called a complex valued metric on $X$ and $(X, d)$ is called a complex valued metric space.

A point $x \in X$ is called an interior point of a set $A \subseteq X$ whenever there exists $\theta \prec r \in \mathbb{C}$ such that $B(x, r)=\{y \in$ $X: d(x, y) \prec r\} \subseteq A$. A subset $A$ in $X$ is called open whenever each point of $A$ is an interior point of $A$. The family $F=\{B(x, r): x \in X, \theta \prec r\}$ is a subbasis for a Hausdorff topology $\tau$ on $X$.

A point $x \in X$ is called a limit point of $A$ whenever, for every $\theta \prec r \in \mathbb{C}, B(x, r) \cap(A \backslash x) \neq \phi$. A subset $B \subseteq X$ is called closed whenever each limit point of $B$ belongs to $B$.
Let $\left\{x_{n}\right\}$ be a sequence in $X$ and $x \in X$. If, for every $c \in \mathbb{C}$, with $\theta \prec c$ there is $n_{0} \in \mathbb{N}$ such that, for all $n>n_{0}, d\left(x_{n}, x\right) \prec c$, then $x$ is called the limit of $\left\{x_{n}\right\}$ and we write $\lim _{n \rightarrow \infty} x_{n}=x$ or $x_{n} \rightarrow x$ as $n \rightarrow \infty$. If for every $c \in \mathbb{C}$, with $\theta \prec c$, there is $n_{0} \in \mathbb{N}$ such that, for all $n>n_{0}, d\left(x_{n}, x_{n+m}\right) \prec c$, then $\left\{x_{n}\right\}$ is called a Cauchy sequence in $(X, d)$. If every Cauchy sequence is convergent in $(X, d)$, then $(X, d)$ is called a complete complex valued metric space.

Lemma 2 (see [18]). Let $(X, d)$ be a complex valued metric space and let $\left\{x_{n}\right\}$ be a sequence in $X$. Then $\left\{x_{n}\right\}$ converges to $x$ if and only if $\left|d\left(x_{n}, x\right)\right| \rightarrow 0$ as $n \rightarrow \infty$.

Lemma 3. Let $(X, d)$ be a complex valued metric space and let $\left\{x_{n}\right\}$ be a sequence in $X$. Then $\left\{x_{n}\right\}$ is a Cauchy sequence if and only if $\sup _{m \geq 1}\left|d\left(x_{n}, x_{n+m}\right)\right| \rightarrow 0$ as $n \rightarrow \infty$.

Recall that if $f$ and $g$ are two self-maps on a set $X$ and $u=f x=g x$ for some $x$ in $X$, then $x$ is called a coincidence point of $f$ and $g$ and $u$ is called a point of coincidence of $f$ and g.

Definition 4 (see [27]). Two self-maps, $f$ and $g$, on a nonempty set $X$ are called weakly compatible if they commute at their coincidence point.

We will also need the following proposition from [27].

Proposition 5. Let $f$ and $g$ be weakly compatible self-maps on $a$ set $X$. If $f$ and $g$ have a unique point of coincidence$w=f x=g x$-then $w$ is the unique common fixed point of $f$ and $g$.

Definition 6 (see [12]). Let $\left\{X_{i}: i=1,2, \ldots, p\right\}$ be a finite collection of nonempty subsets of a set $X$, where $p$ is some positive integer and $f, g: X \rightarrow X$. The set $X$ is said to have a cocyclic representation with respect to the collection $\left\{X_{i}\right.$ : $i=1,2, \ldots, p\}$ and a pair $(f, g)$ if

$$
\begin{aligned}
& \text { (1) } X=\cup_{i=1}^{p} X_{i}, \\
& \text { (2) } f\left(X_{1}\right) \subseteq g\left(X_{2}\right), \ldots, f\left(X_{p-1}\right) \subseteq g\left(X_{p}\right), f\left(X_{p}\right) \subseteq \\
& g\left(X_{1}\right) .
\end{aligned}
$$

Recall that a function $f: \mathbb{C} \rightarrow \mathbb{C}$ is said to be lower semicontinuous at $z_{0}$ in $\mathbb{C}$ if for every $\varepsilon>\theta$ there exists a neighborhood $U$ of $z_{0}$ such that $f(z) \succeq f\left(z_{0}\right)-\varepsilon$ for all $z$ in $U$. This can be expressed as $\liminf _{z \rightarrow z_{0}} f(z) \geq f\left(z_{0}\right)$. Also $f$ is upper semicontinuous at $z_{0}$ in $\mathbb{C}$ if for every $\varepsilon>\theta$ there exists a neighborhood $U$ of $z_{0}$ such that $f(z) \preceq f\left(z_{0}\right)+\varepsilon$ for all $z$ in $U$. This can be expressed as $\lim _{\sup _{z \rightarrow z_{0}}} f(z) \preceq f\left(z_{0}\right)$.

Definition 7. The control functions $\psi$ and $\varphi$ are defined as follows:

(i) $\psi: \mathbb{C}^{+} \rightarrow \mathbb{C}^{+}$is a continuous nondecreasing function with $\psi(z)=\theta$ if and only if $z=\theta$.

(ii) $\varphi: \mathbb{C}^{+} \rightarrow \mathbb{C}^{+}$is a lower semicontinuous function with $\varphi(z)=\theta$ if and only if $z=\theta$. 


\section{Main Results}

Now, we prove fixed and common fixed point theorems in the setup of complex valued metric space. Our first result is the following.

Theorem 8. Let $(X, d)$ be a complete complex valued metric space and $f: X \rightarrow X$. Suppose that there exist control functions $\psi$ and $\varphi$ such that, for $x, y \in X$,

$$
\psi(d(f x, f y)) \preceq \psi\left(U_{f}(x, y)\right)-\varphi\left(U_{f}(x, y)\right)
$$

holds, where

$$
\begin{aligned}
& U_{f}(x, y) \in\left\{d(x, y), \frac{d(f x, x) d(f y, y)}{1+d(x, y)},\right. \\
& \left.\frac{d(f x, y) d(f y, y)}{1+d(x, y)}, \frac{d(f x, y) d(f y, x)}{1+d(x, y)}\right\} .
\end{aligned}
$$

Then $f$ has almost one fixed point.

Proof. Let $x_{0}$ be an arbitrary element in $X$. Construct a sequence $\left\{x_{n}\right\}$ in $X$ as $x_{n+1}=f x_{n}$ or equivalently as $x_{n}=f^{n} x_{0}$ for $n \in \mathbb{N}$. If, for some $n \in \mathbb{N}, x_{n}=x_{n+1}$ holds, then we have $f x_{n}=x_{n+1}=x_{n}$ and $x_{n}$ is the fixed point of $f$. So we assume that $x_{n+1} \neq x_{n}$, for all $n \in \mathbb{N}$; that is, $d\left(x_{n+1}, x_{n}\right)>\theta$ for all $n \in \mathbb{N}$. From (1), we have

$$
\begin{aligned}
\psi\left(d\left(x_{n+1}, x_{n+2}\right)\right)= & \psi\left(d\left(f x_{n}, f x_{n+1}\right)\right) \\
\preceq & \psi\left(U_{f}\left(x_{n}, x_{n+1}\right)\right) \\
& -\varphi\left(U_{f}\left(x_{n}, x_{n+1}\right)\right),
\end{aligned}
$$

where

$$
\begin{aligned}
& U_{f}\left(x_{n}, x_{n+1}\right) \in\left\{d\left(x_{n}, x_{n+1}\right),\right. \\
& \frac{d\left(f x_{n}, x_{n}\right) d\left(f x_{n+1}, x_{n+1}\right)}{1+d\left(x_{n}, x_{n+1}\right)}, \\
& \frac{d\left(f x_{n}, x_{n+1}\right) d\left(f x_{n+1}, x_{n+1}\right)}{1+d\left(x_{n}, x_{n+1}\right)}, \\
& \left.\frac{d\left(f x_{n}, x_{n+1}\right) d\left(f x_{n+1}, x_{n}\right)}{1+d\left(x_{n}, x_{n+1}\right)}\right\}=\left\{d\left(x_{n}, x_{n+1}\right),\right. \\
& \frac{d\left(x_{n+1}, x_{n}\right) d\left(x_{n+2}, x_{n+1}\right)}{1+d\left(x_{n}, x_{n+1}\right)}, \\
& \frac{d\left(x_{n+1}, x_{n+1}\right) d\left(x_{n+2}, x_{n+1}\right)}{1+d\left(x_{n}, x_{n+1}\right)}, \\
& \left.\frac{d\left(x_{n+1}, x_{n+1}\right) d\left(x_{n+2}, x_{n}\right)}{1+d\left(x_{n+1}, x_{n}\right)}\right\}=\left\{d\left(x_{n}, x_{n+1}\right)\right. \\
& \left.\frac{d\left(x_{n+1}, x_{n}\right) d\left(x_{n+2}, x_{n+1}\right)}{1+d\left(x_{n}, x_{n+1}\right)}, \theta, \theta\right\} .
\end{aligned}
$$

$$
\begin{aligned}
& \text { If } U_{f}\left(x_{n}, x_{n+1}\right)=d\left(x_{n}, x_{n+1}\right) \text {, then } \\
& \begin{aligned}
\psi\left(d\left(x_{n+1}, x_{n+2}\right)\right) & \preceq \psi\left(d\left(x_{n}, x_{n+1}\right)\right)-\varphi\left(d\left(x_{n}, x_{n+1}\right)\right) \\
& \preceq \psi\left(d\left(x_{n}, x_{n+1}\right)\right),
\end{aligned}
\end{aligned}
$$

and, by the property of $\psi$, we obtain $d\left(x_{n+1}, x_{n+2}\right) \preceq$ $d\left(x_{n}, x_{n+1}\right)$.

In case $U_{f}\left(x_{n}, x_{n+1}\right)=d\left(x_{n+1}, x_{n}\right) d\left(x_{n+2}, x_{n+1}\right) /(1+$ $\left.d\left(x_{n}, x_{n+1}\right)\right)$, then

$$
\begin{aligned}
\psi\left(d\left(x_{n+1}, x_{n+2}\right)\right) \\
\preceq \psi\left(\frac{d\left(x_{n+1}, x_{n}\right) d\left(x_{n+2}, x_{n+1}\right)}{1+d\left(x_{n}, x_{n+1}\right)}\right) \\
-\varphi\left(\frac{d\left(x_{n+1}, x_{n}\right) d\left(x_{n+2}, x_{n+1}\right)}{1+d\left(x_{n}, x_{n+1}\right)}\right) \\
\preceq \psi\left(d\left(x_{n+2}, x_{n+1}\right)\right) \\
-\varphi\left(\frac{d\left(x_{n+1}, x_{n}\right) d\left(x_{n+2}, x_{n+1}\right)}{1+d\left(x_{n}, x_{n+1}\right)}\right)
\end{aligned}
$$

implies $\varphi\left(d\left(x_{n+1}, x_{n}\right) d\left(x_{n+2}, x_{n+1}\right) /\left(1+d\left(x_{n}, x_{n+1}\right)\right)\right) \preceq \theta$, a contradiction.

Finally if $U_{f}\left(x_{n}, x_{n+1}\right)=\theta$, then $\psi\left(d\left(x_{n+1}, x_{n+2}\right)\right) \preceq \theta$ gives a contradiction.

Thus

$$
d\left(x_{n+1}, x_{n+2}\right) \preceq d\left(x_{n}, x_{n+1}\right)
$$

for all $n \in \mathbb{N}$ and $\left\{d\left(x_{n}, x_{n+1}\right)\right\}$ is the decreasing of positive real numbers. Consequently, there exists $\theta \preceq c \in \mathbb{C}^{+}$such that $\left\{d\left(x_{n}, x_{n+1}\right)\right\}$ converges to $c$. Suppose that $c>\theta$. Now

$$
\begin{aligned}
\psi\left(d\left(x_{n+1}, x_{n+2}\right)\right) \preceq & \psi\left(U_{f}\left(x_{n}, x_{n+1}\right)\right) \\
& -\varphi\left(U_{f}\left(x_{n}, x_{n+1}\right)\right),
\end{aligned}
$$

where $U_{f}\left(x_{n}, x_{n+1}\right) \in\left\{d\left(x_{n}, x_{n+1}\right), d\left(x_{n+1}, x_{n}\right) d\left(x_{n+2}, x_{n+1}\right) /\right.$ $\left.\left(1+d\left(x_{n}, x_{n+1}\right)\right), \theta\right\}$.

If $U_{f}\left(x_{n}, x_{n+1}\right)=d\left(x_{n}, x_{n+1}\right)$, then lower semicontinuity of $\varphi$ gives that

$$
\begin{aligned}
& \limsup _{n \rightarrow \infty} \psi\left(d\left(x_{n+1}, x_{n+2}\right)\right) \\
& \leq \limsup _{n \rightarrow \infty} \psi\left(d\left(x_{n}, x_{n+1}\right)\right) \\
& \quad-\liminf _{n \rightarrow \infty} \varphi\left(d\left(x_{n}, x_{n+1}\right)\right),
\end{aligned}
$$

which implies $\psi(c) \preceq \psi(c)-\varphi(c) \prec \psi(c)$, a contradiction as $\varphi(c)>\theta$.

In case $U_{f}\left(x_{n}, x_{n+1}\right)=d\left(x_{n+1}, x_{n}\right) d\left(x_{n+2}, x_{n+1}\right) /(1+$ $\left.d\left(x_{n}, x_{n+1}\right)\right)$, then we obtain

$$
\begin{aligned}
\limsup _{n \rightarrow \infty} \psi\left(d\left(x_{n+1}, x_{n+2}\right)\right) \\
\leq \limsup _{n \rightarrow \infty} \psi\left(\frac{d\left(x_{n+1}, x_{n}\right) d\left(x_{n+2}, x_{n+1}\right)}{1+d\left(x_{n}, x_{n+1}\right)}\right) \\
\quad-\liminf _{n \rightarrow \infty} \varphi\left(\frac{d\left(x_{n+1}, x_{n}\right) d\left(x_{n+2}, x_{n+1}\right)}{1+d\left(x_{n}, x_{n+1}\right)}\right),
\end{aligned}
$$


which implies that $\psi(c) \leq \psi\left(c^{2} /(1+c)\right)-\varphi\left(c^{2} /(1+c)\right) \prec$ $\psi\left(c^{2} /(1+c)\right)$, and so $c \preceq\left(c^{2} /(1+c)\right)$ which implies $1+c \preceq c$, a contradiction.

If $U_{f}\left(x_{n}, x_{n+1}\right)=\theta$, then we have $\lim \sup _{n \rightarrow \infty} \psi\left(d\left(x_{n+1}\right.\right.$, $\left.\left.x_{n+2}\right)\right) \leq \theta$, a contradiction.

Therefore $c=\theta$; that is, $\lim _{n \rightarrow \infty} d\left(x_{n}, x_{n+1}\right)=\theta$.

Now, we claim that $\lim _{n, m \rightarrow \infty} d\left(x_{n}, x_{m}\right)=\theta$. If not, then there exist $\theta \prec \varepsilon \in \mathbb{C}^{+}$and sequences $\left\{n_{k}\right\},\left\{m_{k}\right\}$ in $\mathbb{N}$, with $n_{k}>m_{k} \geq k$, such that $d\left(x_{n_{k}}, x_{m_{k}}\right) \geq \varepsilon$ for all $k \in \mathbb{N}$. Without any loss of generality, we can assume that $d\left(x_{n_{k}}, x_{m_{k}-1}\right) \prec \varepsilon$. Since $\left\{d\left(x_{m_{k}-1}, x_{m_{k}}\right)\right\}$ is a subsequence of convergent sequence $\left\{d\left(x_{n-1}, x_{n}\right)\right\} \rightarrow \theta$ as $n \rightarrow \infty$, therefore, $\left\{d\left(x_{m_{k}-1}, x_{m_{k}}\right)\right\} \rightarrow \theta$ as $k \rightarrow \infty$. Now

$$
\varepsilon \leq d\left(x_{m_{k}}, x_{n_{k}}\right) \leq d\left(x_{n_{k}}, x_{m_{k}-1}\right)+d\left(x_{m_{k}-1}, x_{m_{k}}\right)
$$

implies that

$$
\lim _{k \rightarrow \infty} d\left(x_{m_{k}}, x_{n_{k}}\right)=\varepsilon
$$

From (12) and inequality $d\left(x_{m_{k}}, x_{n_{k}}\right) \leq d\left(x_{m_{k}}, x_{m_{k}-1}\right)+$ $d\left(x_{m_{k}-1}, x_{n_{k}}\right)$, it follows that $\varepsilon \leq \lim _{k \rightarrow \infty} d\left(x_{m_{k}-1}, x_{n_{k}}\right)$. Also, the inequality $d\left(x_{m_{k}-1}, x_{n_{k}}\right) \leq d\left(x_{m_{k}-1}, x_{m_{k}}\right)+d\left(x_{m_{k}}, x_{n_{k}}\right)$ and (12) give that $\lim _{k \rightarrow \infty} d\left(x_{m_{k}-1}, x_{n_{k}}\right) \leq \varepsilon$, and hence we have

$$
\lim _{k \rightarrow \infty} d\left(x_{m_{k}-1}, x_{n_{k}}\right)=\varepsilon
$$

From (13) and inequality $d\left(x_{m_{k}-1}, x_{n_{k}}\right) \leq d\left(x_{m_{k}-1}, x_{n_{k}+1}\right)+$ $d\left(x_{n_{k}+1}, x_{n_{k}}\right)$, it is implied that $\varepsilon \leq \lim _{k \rightarrow \infty} d\left(x_{m_{k}-1}, x_{n_{k}+1}\right)$, while inequality $d\left(x_{m_{k}-1}, x_{n_{k}+1}\right) \leq d\left(x_{m_{k}-1}, x_{n_{k}}\right)+d\left(x_{n_{k}}\right.$, $\left.x_{n_{k}+1}\right)$ and (13) imply that $\lim _{k \rightarrow \infty} d\left(x_{m_{k}-1}, x_{n_{k}+1}\right) \leq \varepsilon$, and hence we have

$$
\lim _{k \rightarrow \infty} d\left(x_{m_{k}-1}, x_{n_{k}+1}\right)=\varepsilon
$$

From (12) and $d\left(x_{m_{k}}, x_{n_{k}}\right) \leq d\left(x_{m_{k}}, x_{n_{k}+1}\right)+d\left(x_{n_{k}+1}, x_{n_{k}}\right)$, we have $\varepsilon \preceq \lim _{k \rightarrow \infty} d\left(x_{m_{k}}, x_{n_{k}+1}\right)$, and the inequality $d\left(x_{m_{k}}, x_{n_{k}+1}\right) \leq d\left(x_{m_{k}}, x_{n_{k}}\right)+d\left(x_{n_{k}}, x_{n_{k}+1}\right)$ and (12) give that $\lim _{k \rightarrow \infty} d\left(x_{m_{k}}, x_{n_{k}+1}\right) \leq \varepsilon$. So

$$
\lim _{k \rightarrow \infty} d\left(x_{m_{k}}, x_{n_{k}+1}\right)=\varepsilon .
$$

As

$$
\begin{gathered}
M_{f}\left(x_{n_{k}}, x_{m_{k}-1}\right) \in\left\{d\left(x_{n_{k}}, x_{m_{k}-1}\right),\right. \\
\frac{d\left(f x_{n_{k}}, x_{n_{k}}\right) d\left(f x_{m_{k}-1}, x_{m_{k}-1}\right)}{1+d\left(x_{n_{k}}, x_{m_{k}-1}\right)}, \\
\frac{d\left(f x_{n_{k}}, x_{m_{k}-1}\right) d\left(f x_{m_{k}-1}, x_{m_{k}-1}\right)}{1+d\left(x_{n_{k}}, x_{m_{k}-1}\right)},
\end{gathered}
$$

$$
\begin{aligned}
& \left.\frac{d\left(f x_{n_{k}}, x_{m_{k}-1}\right) d\left(f x_{m_{k}-1}, x_{n_{k}}\right)}{1+d\left(x_{n_{k}}, x_{m_{k}-1}\right)}\right\} \\
& =\left\{d\left(x_{n_{k}}, x_{m_{k}-1}\right), \frac{d\left(x_{n_{k}+1}, x_{n_{k}}\right) d\left(x_{m_{k}}, x_{m_{k}-1}\right)}{1+d\left(x_{n_{k}}, x_{m_{k}-1}\right)},\right. \\
& \frac{d\left(x_{n_{k}+1}, x_{m_{k}-1}\right) d\left(x_{m_{k}}, x_{m_{k}-1}\right)}{1+d\left(x_{n_{k}}, x_{m_{k}-1}\right)}, \\
& \left.\frac{d\left(x_{n_{k}+1}, x_{m_{k}-1}\right) d\left(x_{m_{k}}, x_{n_{k}}\right)}{1+d\left(x_{n_{k}}, x_{m_{k}-1}\right)}\right\},
\end{aligned}
$$

so we have

$$
\lim _{k \rightarrow \infty} U_{f}\left(x_{n_{k}}, x_{m_{k}-1}\right) \in\left\{\varepsilon, \theta, \theta, \frac{\varepsilon^{2}}{1+\varepsilon}\right\} .
$$

Now, if $\lim _{k \rightarrow \infty} U_{f}\left(x_{n_{k}}, x_{m_{k}-1}\right)=\varepsilon$, then, from (1), it follows that

$$
\begin{aligned}
\psi\left(d\left(x_{n_{k}+1}, x_{m_{k}}\right)\right)= & \psi\left(d\left(f x_{n_{k}}, f x_{m_{k}-1}\right)\right) \\
\preceq & \psi\left(U_{f}\left(x_{n_{k}}, x_{m_{k}-1}\right)\right) \\
& -\varphi\left(U_{f}\left(x_{n_{k}}, x_{m_{k}-1}\right)\right)
\end{aligned}
$$

and taking upper limit as $k \rightarrow \infty$ implies that $\psi(\varepsilon) \preceq \psi(\varepsilon)-$ $\varphi(\varepsilon) \prec \psi(\varepsilon)$, a contradiction as $\varepsilon>\theta$.

In case $\lim _{k \rightarrow \infty} U_{f}\left(x_{n_{k}}, x_{m_{k}-1}\right)=\theta$, then, from (1), it follows that $\lim \sup _{k \rightarrow \infty} \psi\left(d\left(x_{n_{k}+1}, x_{m_{k}}\right) \leq \theta\right.$, a contradiction.

When $\lim _{k \rightarrow \infty} U_{f}\left(x_{n_{k}}, x_{m_{k}-1}\right)=\varepsilon^{2} /(1+\varepsilon)$, then, from (1), it follows that

$$
\begin{aligned}
\psi(\varepsilon)= & \limsup _{k \rightarrow \infty} \psi\left(d\left(x_{n_{k}+1}, x_{m_{k}}\right)\right) \\
= & \limsup _{k \rightarrow \infty} \psi\left(d\left(f x_{n_{k}}, f x_{m_{k}-1}\right)\right) \\
\leq & \limsup _{k \rightarrow \infty} \psi\left(U_{f}\left(x_{n_{k}}, x_{m_{k}-1}\right)\right) \\
& -\liminf _{k \rightarrow \infty} \varphi\left(U_{f}\left(x_{n_{k}}, x_{m_{k}-1}\right)\right) \\
= & \psi\left(\frac{\varepsilon^{2}}{1+\varepsilon}\right)-\varphi\left(\frac{\varepsilon^{2}}{1+\varepsilon}\right) \leq \psi\left(\frac{\varepsilon^{2}}{1+\varepsilon}\right),
\end{aligned}
$$

which implies $\varepsilon \preceq \varepsilon^{2} /(1+\varepsilon)$; that is, $1+\varepsilon \preceq \varepsilon$, a contradiction.

Thus, we obtain that $\lim _{n, m \rightarrow \infty} d\left(x_{n}, x_{m}\right)=\theta$, and hence $\left\{x_{n}\right\}$ is a Cauchy sequence in the complex valued metric space $(X, d)$.

Next, we assume there exists a point $u \in X$ such that $\lim _{n \rightarrow \infty} d\left(u, x_{n}\right)=\theta$. 
Thus,

$$
\begin{aligned}
& U_{f}\left(u, x_{n}\right) \in\left\{d\left(u, x_{n}\right), \frac{d(f u, u) d\left(f x_{n}, x_{n}\right)}{1+d\left(u, x_{n}\right)},\right. \\
& \left.\frac{d\left(f u, x_{n}\right) d\left(f x_{n}, x_{n}\right)}{1+d\left(u, x_{n}\right)}, \frac{d\left(f u, x_{n}\right) d\left(f x_{n}, u\right)}{1+d\left(u, x_{n}\right)}\right\} \\
& =\left\{d\left(u, x_{n}\right), \frac{d(f u, u) d\left(x_{n+1}, x_{n}\right)}{1+d\left(u, x_{n}\right)},\right. \\
& \left.\frac{d\left(f u, x_{n}\right) d\left(x_{n+1}, x_{n}\right)}{1+d\left(u, x_{n}\right)}, \frac{d\left(f u, x_{n}\right) d\left(x_{n+1}, u\right)}{1+d\left(u, x_{n}\right)}\right\} .
\end{aligned}
$$

Taking limit as $n \rightarrow \infty$, we conclude that $\lim _{n \rightarrow \infty} M_{f}(u$, $\left.x_{n}\right)=\theta$. Hence

$$
\psi\left(d\left(f u, f x_{n+1}\right)\right) \preceq \psi\left(U_{f}\left(u, x_{n}\right)\right)-\varphi\left(U_{f}\left(u, x_{n}\right)\right)
$$

and taking upper limit as $n \rightarrow \infty$ implies that

$$
\psi(d(f u, u)) \preceq \theta,
$$

which further implies that $f u=u$.

To prove the uniqueness of fixed point of $f$, assume on the contrary that $f v=v$ and $f w=w$ with $v \neq w$. Note that

$$
\begin{aligned}
\psi(d(v, w)) & =\psi(d(f v, f w)) \\
& \preceq \psi\left(U_{f}(v, w)\right)-\varphi\left(U_{f}(v, w)\right),
\end{aligned}
$$

where

$$
\begin{aligned}
& U_{f}(v, w) \in\left\{d(v, w), \frac{d(f v, v) d(f w, w)}{1+d(v, w)},\right. \\
& \left.\frac{d(f v, w) d(f w, w)}{1+d(v, w)}, \frac{d(f v, w) d(f w, v)}{1+d(v, w)}\right\} \\
& =\left\{d(v, w), \frac{d(v, v) d(w, w)}{1+d(v, w)}, \frac{d(v, w) d(w, w)}{1+d(v, w)},\right. \\
& \left.\frac{d(v, w) d(w, v)}{1+d(v, w)}\right\}=\left\{d(v, w), \theta, \theta, \frac{d(v, w)^{2}}{1+d(v, w)}\right\} .
\end{aligned}
$$

Now, if $U_{f}(v, w)=d(v, w)$, then from (23) it follows that

$$
\begin{aligned}
\psi(d(v, w)) & =\psi(d(f v, f w)) \\
& \preceq \psi(d(v, w))-\varphi(d(v, w)) \\
& \prec \psi(d(v, w))
\end{aligned}
$$

a contradiction as $d(v, w)>\theta$. Hence $v=w$.

In case $U_{f}(v, w)=\theta$, then (23) gives a contradiction. (23),

Finally, if $U_{f}(v, w)=d(v, w)^{2} /(1+d(v, w))$, then, from

$$
\begin{aligned}
\psi(d(v, w)) & \preceq \psi\left(\frac{d(v, w)^{2}}{1+d(v, w)}\right)-\varphi\left(\frac{d(v, w)^{2}}{1+d(v, w)}\right) \\
& \prec \psi\left(\frac{d(v, w)^{2}}{1+d(v, w)}\right),
\end{aligned}
$$

which gives $1+d(v, w) \preceq d(v, w)$, a contradiction. Hence for all cases we have $v=w$.

Corollary 9. Let $(X, d)$ be a complete complex valued metric space and $f: X \rightarrow X$. Suppose that there exist control functions $\psi$ and $\varphi$ such that for $x, y \in X$

$$
\psi\left(d\left(f^{n} x, f^{n} y\right)\right) \preceq \frac{\psi\left(U_{f^{n}}(x, y)\right)}{\varphi\left(U_{f^{n}}(x, y)\right)}
$$

holds for $n \in \mathbb{N}$, where

$$
\begin{aligned}
& U_{f^{n}}(x, y) \in\left\{d(x, y), \frac{d\left(f^{n} x, x\right) d\left(f^{n} y, y\right)}{1+d(x, y)},\right. \\
& \left.\frac{d\left(f^{n} x, y\right) d\left(f^{n} y, y\right)}{1+d(x, y)}, \frac{d\left(f^{n} x, y\right) d\left(f^{n} y, x\right)}{1+d(x, y)}\right\} .
\end{aligned}
$$

Then $f$ has almost one fixed point $u$ in $X$ and $f^{n} u=f u$.

Proof. Taking $T=f^{n}$. It follows from Theorem 8 that $T$ has a unique fixed point $u$. Also $f(u)=f(T u)=f^{n+1}(u)=$ $f^{n}(f u)=T(f u)$ implies that $f u$ is also the fixed point of $T$. From the uniqueness of fixed point of $T$, it is implied that $f u=u$.

Recently, Haghi et al. [28] proved the following lemma.

Lemma 10. Let $X$ be a nonempty set and let $f$ be a self-map on $X$. Then there exists a subset $E \subseteq X$ such that $f: E \rightarrow X$ is one-to-one and $f(E)=f(X)$.

Theorem 11. Let $(X, d)$ be a complex valued metric space and $f, g: X \rightarrow X$. Suppose that there exist control functions $\psi$ and $\varphi$ such that for any $x, y \in X$

$$
\psi(d(f x, f y)) \preceq \psi(U(g x, g y))-\varphi(U(g x, g y))
$$

holds, where

$$
\begin{gathered}
U(x, y) \in\left\{d(g x, g y), \frac{d(f x, g x) d(f y, g y)}{1+d(g x, g y)},\right. \\
\left.\frac{d(f x, g y) d(f y, g y)}{1+d(g x, g y)}, \frac{d(f x, g y) d(f y, g x)}{1+d(g x, g y)}\right\} .
\end{gathered}
$$

If $g(X)$ is a complete subspace of $X$, then $f$ and $g$ have at most one coincidence point in $X$. Furthermore, if $f$ and $g$ are weakly compatible, then $f$ and $g$ have a unique common fixed point in $X$.

Proof. It follows from Lemma 10 that there exists $E \subseteq X$ such that $g: E \rightarrow X$ is one-to-one and $g(E)=g(X)$. Define a map $h: g(E) \rightarrow g(E)$ by $h(g x)=f x$. Since $g$ is one-to-one on $E, h$ is well-defined. Note that

$$
\begin{aligned}
& \psi(d(h(g x), h(g y))) \\
& \quad \leq \psi(U(g x, g y))-\varphi(U(g x, g y)),
\end{aligned}
$$


for all $g x, g y \in g(E)$, where

$$
\begin{aligned}
& U(g x, g y) \in\{d(g x, g y), \\
& \frac{d(h(g x), g x) d(h(g y), g y)}{1+d(g x, g y)}, \\
& \frac{d(h(g x), g y) d(h(g y), g y)}{1+d(g x, g y)}, \\
& \left.\frac{d(h(g x), g y) d(h(g y), g x)}{1+d(g x, g y)}\right\} .
\end{aligned}
$$

Since $g(E)=g(X)$ is complete, by Theorem 8 , there exists $x^{*} \in X$ such that $h\left(g x^{*}\right)=g x^{*}=f x^{*}$. Hence, $f$ and $g$ have at most one point of coincidence in $X$. By Proposition 5, $f$ and $g$ have at most one common fixed point.

\section{Cocyclic Contraction Mappings}

In this section, we prove common fixed point results for selfmaps satisfying cocyclic contractions defined on a complex valued metric space. Our first result is the following.

Theorem 12. Let $(X, d)$ be a complex valued metric space, let $A_{1}, A_{2}, \ldots, A_{p}$ be nonempty closed subsets of $X$, and let $Y=$ $\cup_{i=1}^{p} A_{i}$. Suppose that $f, g: Y \rightarrow Y$ are such that

(a) $Y$ has a cocyclic representation with respect to pair $(f, g)$ and to the collection $\left\{A_{i}: i=1,2, \ldots, p\right\}$;

(b) there exist control functions $\psi$ and $\varphi$ such that, for any $(x, y) \in A_{i} \times A_{i+1}, i=1,2, \ldots, p$,

$$
\psi(d(f x, f y)) \preceq \psi\left(U_{f, g}(x, y)\right)-\varphi\left(U_{f, g}(x, y)\right)
$$

holds where

$$
\begin{aligned}
& U_{f, g}(x, y) \in\left\{d(g x, g y), \frac{d(f x, g x) d(f y, g y)}{1+d(g x, g y)},\right. \\
& \left.\frac{d(f x, g y) d(f y, g y)}{1+d(g x, g y)}, \frac{d(f x, g y) d(f y, g x)}{1+d(g x, g y)}\right\}, \\
& \text { with } A_{p+1}=A_{1} .
\end{aligned}
$$

If $g\left(A_{i}\right)$ is complete subspace of $X$ for each $i \in\{1,2, \ldots, p\}$, then $f$ and $g$ have a unique coincidence point $z \in \cap_{i=1}^{p} g\left(A_{i}\right)=$ $K$ provided that $f(K) \subseteq g(K) \subseteq K$ and $g(K)$ is closed. Furthermore, if $f$ and $g$ are weakly compatible, then $f$ and $g$ have a unique common fixed point.

Proof. Let $x_{0} \in \cup_{i=1}^{p} A_{i}$ be a given element. By the given condition, there exists $i_{0} \in\{1,2, \ldots, p\}$ such that $x_{0} \in A_{i_{0}}$. Since $f\left(A_{i_{0}}\right) \subseteq g\left(A_{i_{0}+1}\right)$, we can choose a point $x_{1} \in A_{i_{0}+1}$ such that $f\left(x_{0}\right)=g\left(x_{1}\right)$. Continuing this process, for $n>0$, there exists $i_{n} \in\{1,2, \ldots, p\}$ such that, having chosen $x_{n} \in$ $A_{i_{n}}$, we obtain $x_{n+1} \in A_{i_{n}+1}$ such that $f\left(x_{n}\right)=g\left(x_{n+1}\right)$.
If for some $n_{0} \geq 0, f\left(x_{n_{0}}\right)=f\left(x_{n_{0}+1}\right)$; that is, $f\left(x_{n_{0}}\right)=$ $g\left(x_{n_{0}}\right)$ and $x_{n_{0}}$ is the coincidence point of $f$ and $g$. Taking $d\left(f\left(x_{n}\right), f\left(x_{n+1}\right)\right)>\theta$ for all $n$, we have

$$
\begin{aligned}
\psi\left(d\left(g x_{n+1}, g x_{n+2}\right)\right)= & \psi\left(d\left(f x_{n}, f x_{n+1}\right)\right) \\
\preceq & \psi\left(U_{f, g}\left(x_{n}, x_{n+1}\right)\right) \\
& -\varphi\left(U_{f, g}\left(x_{n}, x_{n+1}\right)\right) \\
& \preceq \psi\left(U_{f, g}\left(x_{n}, x_{n+1}\right)\right),
\end{aligned}
$$

where

$$
\begin{aligned}
& U_{f, g}\left(x_{n}, x_{n+1}\right) \in\left\{d\left(g x_{n}, g x_{n+1}\right),\right. \\
& \frac{d\left(f x_{n}, g x_{n}\right) d\left(f x_{n+1}, g x_{n+1}\right)}{1+d\left(g x_{n}, g x_{n+1}\right)}, \\
& \quad \frac{d\left(f x_{n}, g x_{n+1}\right) d\left(f x_{n+1}, g x_{n+1}\right)}{1+d\left(g x_{n}, g x_{n+1}\right)}, \\
& \left.\frac{d\left(f x_{n}, g x_{n+1}\right) d\left(f x_{n+1}, g x_{n}\right)}{1+d\left(g x_{n}, g x_{n+1}\right)}\right\}=\left\{d\left(g x_{n}, g x_{n+1}\right),\right. \\
& \frac{d\left(g x_{n+1}, g x_{n}\right) d\left(g x_{n+2}, g x_{n+1}\right)}{1+d\left(g x_{n}, g x_{n+1}\right)}, \\
& \frac{d\left(g x_{n+1}, g x_{n+1}\right) d\left(g x_{n+2}, g x_{n+1}\right)}{1+d\left(g x_{n}, g x_{n+1}\right)^{2}}, \\
& \left.\quad \frac{d\left(g x_{n+1}, g x_{n+1}\right) d\left(g x_{n+2}, g x_{n}\right)}{1+d\left(g x_{n}, g x_{n+1}\right)}\right\} \\
& \quad\left\{d\left(g x_{n}, g x_{n+1}\right),\right. \\
& \left.\frac{d\left(g x_{n+1}, g x_{n}\right) d\left(g x_{n+2}, g x_{n+1}\right)}{1+d\left(g x_{n}, g x_{n+1}\right)}, \theta, \theta\right\} .
\end{aligned}
$$

If, for some $n, U_{f, g}\left(x_{n}, x_{n+1}\right)=d\left(g x_{n}, g x_{n+1}\right)$, then

$$
\begin{aligned}
\psi\left(d\left(g x_{n+1}, g x_{n+2}\right)\right) \preceq & \psi\left(d\left(g x_{n}, g x_{n+1}\right)\right) \\
& -\varphi\left(d\left(g x_{n}, g x_{n+1}\right)\right)
\end{aligned}
$$

implies that $d\left(g x_{n+2}, g x_{n+1}\right) \preceq d\left(g x_{n}, g x_{n+1}\right)$. 
In case $U_{f, g}\left(x_{n}, x_{n+1}\right)=d\left(g x_{n+1}, g x_{n}\right) d\left(g x_{n+2}, g x_{n+1}\right) /$ $\left(1+d\left(g x_{n}, g x_{n+1}\right)\right)$, then

$$
\begin{aligned}
\psi( & \left.\left(g x_{n+1}, g x_{n+2}\right)\right) \\
\leq & \psi\left(\frac{d\left(g x_{n+1}, g x_{n}\right) d\left(g x_{n+2}, g x_{n+1}\right)}{1+d\left(g x_{n}, g x_{n+1}\right)}\right) \\
& -\varphi\left(\frac{d\left(g x_{n+1}, g x_{n}\right) d\left(g x_{n+2}, g x_{n+1}\right)}{1+d\left(g x_{n}, g x_{n+1}\right)}\right) \\
\leq & \psi\left(d\left(g x_{n+2}, g x_{n+1}\right)\right) \\
& -\varphi\left(\frac{d\left(g x_{n+1}, g x_{n}\right) d\left(g x_{n+2}, g x_{n+1}\right)}{1+d\left(g x_{n}, g x_{n+1}\right)}\right)
\end{aligned}
$$

implies $\varphi\left(d\left(g x_{n+1}, g x_{n}\right) d\left(g x_{n+2}, g x_{n+1}\right) /\left(1+d\left(g x_{n}\right.\right.\right.$, $\left.\left.\left.g x_{n+1}\right)\right)\right) \preceq \theta$, a contradiction.

When $U_{f, g}\left(x_{n}, x_{n+1}\right)=\theta$, then $\psi\left(d\left(g x_{n+1}, g x_{n+2}\right)\right) \preceq \theta$ gives a contradiction.

Thus

$$
d\left(g x_{n+1}, g x_{n+2}\right) \leq d\left(g x_{n}, g x_{n+1}\right)
$$

for all $n \in \mathbb{N}$ and $\left\{d\left(g x_{n}, g x_{n+1}\right)\right\}$ is the decreasing of positive real numbers. Consequently, there exists $\theta \preceq \delta \in \mathbb{C}^{+}$such that $\left\{d\left(x_{n}, x_{n+1}\right)\right\}$ converges to $\delta$. Suppose that $\delta>\theta$. Now

$$
\begin{aligned}
\psi\left(d\left(g x_{n+1}, g x_{n+2}\right)\right) \leq & \psi\left(U_{f, g}\left(x_{n}, x_{n+1}\right)\right) \\
& -\varphi\left(U_{f, g}\left(x_{n}, x_{n+1}\right)\right),
\end{aligned}
$$

where

$$
\begin{aligned}
& U_{f, g}\left(x_{n}, x_{n+1}\right) \in\left\{d\left(g x_{n}, g x_{n+1}\right),\right. \\
& \left.\quad \frac{d\left(g x_{n+1}, g x_{n}\right) d\left(g x_{n+2}, g x_{n+1}\right)}{1+d\left(g x_{n}, g x_{n+1}\right)}, \theta\right\} .
\end{aligned}
$$

If $U_{f}\left(x_{n}, x_{n+1}\right)=d\left(g x_{n}, g x_{n+1}\right)$, then lower semicontinuity of $\varphi$ gives that

$$
\begin{aligned}
& \limsup _{n \rightarrow \infty} \psi\left(d\left(g x_{n+1}, g x_{n+2}\right)\right) \\
& \leq \limsup _{n \rightarrow \infty} \psi\left(d\left(g x_{n}, g x_{n+1}\right)\right) \\
& \quad-\liminf _{n \rightarrow \infty} \varphi\left(d\left(g x_{n}, g x_{n+1}\right)\right),
\end{aligned}
$$

which implies that $\psi(\delta) \preceq \psi(\delta)-\varphi(\delta) \prec \psi(\delta)$, a contradiction as $\varphi(\delta)>\theta$.

In case $U_{f, g}\left(x_{n}, x_{n+1}\right)=d\left(g x_{n+1}, g x_{n}\right) d\left(g x_{n+2}, g x_{n+1}\right) /$ $\left(1+d\left(g x_{n}, g x_{n+1}\right)\right)$, then we obtain

$$
\begin{aligned}
\limsup _{n \rightarrow \infty} \psi\left(d\left(g x_{n+1}, g x_{n+2}\right)\right) \\
\leq \limsup _{n \rightarrow \infty} \psi\left(\frac{d\left(g x_{n+1}, g x_{n}\right) d\left(g x_{n+2}, g x_{n+1}\right)}{1+d\left(g x_{n}, g x_{n+1}\right)}\right) \\
\quad-\liminf _{n \rightarrow \infty} \varphi\left(\frac{d\left(g x_{n+1}, g x_{n}\right) d\left(g x_{n+2}, g x_{n+1}\right)}{1+d\left(g x_{n}, g x_{n+1}\right)}\right),
\end{aligned}
$$

which implies that $\psi(\delta) \preceq \psi\left(\delta^{2} /(1+\delta)\right)-\varphi\left(\delta^{2} /(1+\delta)\right) \prec$ $\psi\left(\delta^{2} /(1+\delta)\right)$, and so $\delta \preceq \delta^{2} /(1+\delta)$ which implies $1+\delta \preceq \delta$, a contradiction.

If $U_{f, g}\left(x_{n}, x_{n+1}\right)=\theta$, then we have $\psi(\delta)=$ $\lim \sup _{n \rightarrow \infty} \psi\left(d\left(g x_{n+1}, g x_{n+2}\right)\right) \preceq \theta$, a contradiction. Therefore $\delta=\theta$; that is,

$$
\lim _{n \rightarrow \infty} d\left(g x_{n+1}, g x_{n}\right)=\theta .
$$

Now we claim that $\left\{g x_{n}\right\}$ is a complex valued Cauchy sequence. If not, there are $\varepsilon>\theta$ and even integers $n_{k}$ and $m_{k}$ with $m_{k}>n_{k}>k$ such that

$$
d\left(g x_{m_{k}}, g x_{n_{k}}\right) \geq \varepsilon
$$

and $d\left(g x_{m_{k}-1}, g x_{n_{k}}\right)<\varepsilon$. Note that

$$
\begin{aligned}
\varepsilon & \leq d\left(g x_{m_{k}}, g x_{n_{k}}\right) \\
& \leq d\left(g x_{n_{k}}, g x_{m_{k}-1}\right)+d\left(g x_{m_{k}-1}, g x_{m_{k}}\right) .
\end{aligned}
$$

From (44) and (45), it follows that

$$
\lim _{k \rightarrow \infty} d\left(g x_{m_{k}}, g x_{n_{k}}\right)=\varepsilon
$$

By (47) and

$$
\begin{aligned}
d\left(g x_{n_{k}+1}, g x_{m_{k}}\right) \leq & d\left(g x_{n_{k}+1}, g x_{n_{k}}\right) \\
& +d\left(g x_{n_{k}}, g x_{m_{k}}\right),
\end{aligned}
$$

we have $\lim _{k \rightarrow \infty} d\left(g x_{n_{k}+1}, g x_{m_{k}}\right) \leq \varepsilon$. Also, by (47) and

$$
\begin{aligned}
d\left(g x_{m_{k}}, g x_{n_{k}}\right) \preceq & d\left(g x_{n_{k}+1}, g x_{n_{k}}\right) \\
& +d\left(g x_{n_{k}+1}, g x_{m_{k}}\right),
\end{aligned}
$$

we obtain that $\varepsilon \preceq \lim _{k \rightarrow \infty} d\left(g x_{n_{k}+1}, g x_{m_{k}}\right)$. Hence

$$
\lim _{k \rightarrow \infty} d\left(g x_{n_{k}+1}, g x_{m_{k}}\right)=\varepsilon .
$$

From (44) and

$$
\begin{aligned}
d\left(g x_{n_{k+1}}, g x_{m_{k}-1}\right) \leq & d\left(g x_{n_{k}+1}, g x_{n_{k}}\right) \\
& +d\left(g x_{n_{k}}, g x_{m_{k}-1}\right),
\end{aligned}
$$

we have $\lim _{k \rightarrow \infty} d\left(g x_{n_{k}+1}, g x_{m_{k}-1}\right) \leq \varepsilon$. By (50) and the inequality

$$
\begin{aligned}
d\left(g x_{n_{k}+1}, g x_{m_{k}}\right) \leq & d\left(g x_{n_{k}+1}, g x_{m_{k}-1}\right) \\
& +d\left(g x_{m_{k}-1}, g x_{m_{k}}\right),
\end{aligned}
$$

we have $\varepsilon \preceq \lim _{k \rightarrow \infty} d\left(g x_{n_{k}+1}, g x_{m_{k}-1}\right)$. Thus

$$
\lim _{k \rightarrow \infty} d\left(g x_{n_{k}+1}, g x_{m_{k}-1}\right)=\varepsilon .
$$


As

$$
\begin{aligned}
& U_{f, g}\left(x_{n_{k}}, x_{m_{k}-1}\right) \in\left\{d\left(g x_{n_{k}}, g x_{m_{k}-1}\right),\right. \\
& \frac{d\left(f x_{n_{k}}, g x_{n_{k}}\right) d\left(f x_{m_{k}-1}, g x_{m_{k}-1}\right)}{1+d\left(g x_{n_{k}}, g x_{m_{k}-1}\right)}, \\
& \frac{d\left(f x_{n_{k}}, g x_{m_{k}-1}\right) d\left(f x_{m_{k}-1}, g x_{m_{k}-1}\right)}{1+d\left(g x_{n_{k}}, g x_{m_{k}-1}\right)}, \\
& \left.\frac{d\left(f x_{n_{k}}, g x_{m_{k}-1}\right) d\left(f x_{m_{k}-1}, g x_{n_{k}}\right)}{1+d\left(g x_{n_{k}}, g x_{m_{k}-1}\right)}\right\} \\
& =\left\{d\left(g x_{n_{k}}, g x_{m_{k}-1}\right),\right. \\
& \frac{d\left(g x_{n_{k}+1}, g x_{n_{k}}\right) d\left(g x_{m_{k}}, g x_{m_{k}-1}\right)}{1+d\left(g x_{n_{k}}, g x_{m_{k}-1}\right)}, \\
& \frac{d\left(g x_{n_{k}+1}, g x_{m_{k}-1}\right) d\left(g x_{m_{k}}, g x_{m_{k}-1}\right)}{1+d\left(g x_{n_{k}}, g x_{m_{k}-1}\right)}, \\
& \left.\frac{d\left(g x_{n_{k}+1}, g x_{m_{k}-1}\right) d\left(g x_{m_{k}}, g x_{n_{k}}\right)}{1+d\left(g x_{n_{k}}, g x_{m_{k}-1}\right)}\right\},
\end{aligned}
$$

so we have $\lim _{k \rightarrow \infty} U_{f, g}\left(x_{n_{k}}, x_{m_{k}-1}\right) \in\left\{\varepsilon, \theta, \theta, \varepsilon^{2} /(1+\varepsilon)\right\}$.

Now, if $\lim _{k \rightarrow \infty} U_{f, g}\left(x_{n_{k}}, x_{m_{k}-1}\right)=\varepsilon$, then, from (b), it follows that

$$
\begin{aligned}
\psi\left(d\left(g x_{n_{k}+1}, g x_{m_{k}}\right)\right)= & \psi\left(d\left(f x_{n_{k}}, f x_{m_{k}-1}\right)\right) \\
\leq & \psi\left(M_{f, g}\left(x_{n_{k}}, x_{m_{k}-1}\right)\right) \\
& -\varphi\left(M_{f, g}\left(x_{n_{k}}, x_{m_{k}-1}\right)\right)
\end{aligned}
$$

and taking upper limit as $k \rightarrow \infty$ implies that $\psi(\varepsilon) \preceq \psi(\varepsilon)-$ $\varphi(\varepsilon) \prec \psi(\varepsilon)$, a contradiction as $\varepsilon>\theta$.

In case $\lim _{k \rightarrow \infty} M_{f, g}\left(x_{n_{k}}, x_{m_{k}-1}\right)=\theta$, then, from (b), it follows that $\lim \sup _{k \rightarrow \infty} \psi\left(d\left(g x_{n_{k}+1}, g x_{m_{k}}\right)\right) \preceq \theta$, a contradiction.

When $\lim _{k \rightarrow \infty} M_{f, g}\left(x_{n_{k}}, x_{m_{k}-1}\right)=\varepsilon^{2} /(1+\varepsilon)$, then, from (b), it follows that

$$
\begin{aligned}
\psi(\varepsilon)= & \limsup _{k \rightarrow \infty} \psi\left(d\left(g x_{n_{k}+1}, g x_{m_{k}}\right)\right) \\
= & \limsup _{k \rightarrow \infty} \psi\left(d\left(f x_{n_{k}}, f x_{m_{k}-1}\right)\right) \\
\leq & \limsup _{k \rightarrow \infty} \psi\left(U_{f, g}\left(x_{n_{k}}, x_{m_{k}-1}\right)\right) \\
& -\liminf _{k \rightarrow \infty} \varphi\left(U_{f, g}\left(x_{n_{k}}, x_{m_{k}-1}\right)\right) \\
= & \psi\left(\frac{\varepsilon^{2}}{1+\varepsilon}\right)-\varphi\left(\frac{\varepsilon^{2}}{1+\varepsilon}\right) \preceq \psi\left(\frac{\varepsilon^{2}}{1+\varepsilon}\right),
\end{aligned}
$$

which implies $\varepsilon \preceq \varepsilon^{2} /(1+\varepsilon)$; that is, $1+\varepsilon \preceq \varepsilon$, a contradiction.
Hence $\left\{g x_{n}\right\}$ is a Cauchy sequence in $\cup_{i=1}^{p} g\left(A_{i}\right)$. Since $\cup_{i=1}^{p} g\left(A_{i}\right)$ is complete, there exists $y \in \cup_{i=1}^{p} g\left(A_{i}\right)$ such that

$$
\lim _{n \rightarrow \infty} d\left(g x_{n}, y\right)=\theta .
$$

Consequently, we can find a point $z$ in $Y$ such that $g(z)=y$.

Now we show that $y \in \cap_{i=1}^{p} g\left(A_{i}\right)=K$. From condition (a) and $x_{0} \in A_{i_{0}}$ for some $i_{0} \in\{1,2, \ldots, p\}$, we can choose a subsequence $\left\{x_{n_{k}}\right\}$ in $A_{i_{0}}$ out of the sequence $\left\{x_{n}\right\}$. Obviously, $\left\{g x_{n_{k}}\right\} \subseteq g\left(A_{i_{0}}\right)$. As $g\left(A_{i_{0}}\right)$ is closed, so $y \in g\left(A_{i_{0}}\right)$. Similarly, we can choose a subsequence $\left\{x_{n_{k}+1}\right\}$ in $A_{i_{0}+1}$ out of the sequence $\left\{x_{n}\right\}$. Obviously, $\left\{g x_{n_{k}+1}\right\} \subseteq g\left(A_{i_{0}+1}\right)$. As $g\left(A_{i_{0}+1}\right)$ is closed, so $y \in g\left(A_{i_{0}+1}\right)$. Continuing this way, we obtain that $y \in \cap_{i=1}^{p} g\left(A_{i}\right)$ and hence $\cap_{i=1}^{p} g\left(A_{i}\right) \neq \emptyset$.

Now we show that $f(z)=g(z)$. Since $z \in Y$, there exists some $i$ in $\{1,2, \ldots, p\}$ such that $z \in A_{i}$. Choose a subsequence $\left\{x_{n_{k}}\right\}$ of $\left\{x_{n}\right\}$ with $x_{n_{k}} \in A_{i-1}$. From (b), we have

$$
\begin{aligned}
\psi\left(d\left(g x_{n_{k}+1}, f z\right)\right)= & \psi\left(d\left(f x_{n_{k}}, f z\right)\right) \\
\preceq & \psi\left(U_{f, g}\left(x_{n_{k}}, z\right)\right) \\
& -\varphi\left(U_{f, g}\left(x_{n_{k}}, z\right)\right),
\end{aligned}
$$

where

$$
\begin{aligned}
& U_{f, g}\left(x_{n_{k}}, z\right) \in\left\{d\left(g x_{n_{k}}, g z\right),\right. \\
& \frac{d\left(f x_{n_{k}}, g x_{n_{k}}\right) d(f z, g z)}{1+d\left(g x_{n_{k}}, g z\right)}, \frac{d\left(f x_{n_{k}}, g z\right) d(f z, g z)}{1+d\left(g x_{n_{k}}, g z\right)}, \\
& \left.\frac{d\left(f x_{n_{k}}, g z\right) d\left(f z, g x_{n_{k}}\right)}{1+d\left(g x_{n_{k}}, g z\right)}\right\}=\left\{d\left(g x_{n_{k}}, g z\right),\right. \\
& \frac{d\left(g x_{n_{k}+1}, g x_{n_{k}}\right) d(f z, g z)}{1+d\left(g x_{n_{k}}, g z\right)}, \\
& \frac{d\left(g x_{n_{k}+1}, g z\right) d(f z, g z)}{1+d\left(g x_{n_{k}}, g z\right)}, \\
& \left.\frac{d\left(g x_{n_{k}+1}, g z\right) d\left(f z, g x_{n_{k}}\right)}{1+d\left(g x_{n_{k}}, g z\right)}\right\},
\end{aligned}
$$

which gives $\lim _{k \rightarrow \infty} U_{f, g}\left(x_{n_{k}}, z\right)=\theta$. Thus $\psi(d(g z, f z)) \preceq \theta$ and hence $f(z)=g(z)$. Thus $z$ is the coincidence point of $f$ and $g$.

Thus $K$ being a finite intersection of closed sets is closed and hence complete. Consider the restrictions mappings $\left.f\right|_{K},\left.g\right|_{K}: K \rightarrow K$. Clearly, $\left.\left.f\right|_{K}(K) \subseteq g\right|_{K}(K) \subseteq K$. Also $\left.g\right|_{Z}(K)$ is closed and hence complete. From Theorem 11, it follows that $\left.f\right|_{K}$ and $\left.g\right|_{K}$ have at most one coincidence point in $K$. As $f$ and $g$ are weakly compatible, from Proposition 5, it follows that $\left.f\right|_{K}$ and $\left.g\right|_{K}$ have at most one common fixed point. 
Corollary 13. Let $(X, d)$ be a complex valued metric space, let $A_{1}, A_{2}, \ldots, A_{p}$ be nonempty closed subsets of $X$, and let $Y=$ $\cup_{i=1}^{p} A_{i}$. Suppose that $f, g: Y \rightarrow Y$ are such that

(a) $Y$ has a cocyclic representation with respect to pair $\left(f^{m}, g^{n}\right)$ and to the collection $\left\{A_{i}: i=1,2, \ldots, p\right\}$ for some $m, n \in \mathbb{N}$;

(b) there exist two control functions, $\psi$ and $\varphi$, such that, for any $(x, y) \in A_{i} \times A_{i+1}, i=1,2, \ldots, p$,

$$
\psi\left(d\left(f^{m} x, f^{m} y\right)\right) \leq \psi\left(U_{f, g}(x, y)\right)-\varphi\left(U_{f, g}(x, y)\right)
$$

holds for some $m, n \in \mathbb{N}$, where

$$
\begin{aligned}
& U_{f}(x, y) \in\left\{d\left(g^{n} x, g^{n} y\right),\right. \\
& \frac{d\left(f^{m} x, g^{n} x\right) d\left(f^{m} y, g^{n} y\right)}{1+d\left(g^{n} x, g^{n} y\right)}, \\
& \frac{d\left(f^{m} x, g^{n} y\right) d\left(f^{m} y, g^{n} y\right)}{1+d\left(g^{n} x, g^{n} y\right)^{2}}, \\
& \left.\frac{d\left(f^{m} x, g^{n} y\right) d\left(f^{m} y, g^{n} x\right)}{1+d\left(g^{n} x, g^{n} y\right)}\right\},
\end{aligned}
$$

with $A_{p+1}=A_{1}$.

If $g^{n}\left(A_{i}\right)$ is a complete subspace of $X$ for each $i \in\{1,2, \ldots, p\}$, then $f^{m}$ and $g^{n}$ have a unique coincidence point $z \in$ $\cap_{i=1}^{p} g^{n}\left(A_{i}\right)=K$ provided that $f^{m}(K) \subseteq g^{n}(K) \subseteq K$ and $g^{n}(K)$ is closed. Moreover, if $f^{m}$ and $g^{n}$ are weakly compatible, then $f^{m}$ and $g^{n}$ have a unique common fixed point. Furthermore, $f$ and $g$ have at most one common fixed point provided that $f$ and $g$ commute.

Proof. Set $F=f^{m}$ and $G=g^{n}$. From Theorem 12, $F$ and $G$ have a unique common fixed point $u$. Now $F(f u)=f(F u)=$ $f u$ and $G(f u)=f(G u)=f u$ imply that $f u$ is the common fixed point of $F$ and $G$. Also, $G(g u)=g(G u)=g u$ and $F(g u)=g(F u)=g u$ imply that $g u$ is also the common fixed point of $F$ and $G$. By the uniqueness of common fixed point of $F$ and $G$, we have $f u=g u=u$.

Example 14. Let $X=\mathbb{R}$ and let $d: X \times X \rightarrow \mathbb{C}^{+}$be the complex valued metric defined by $d(x, y)=|x-y| e^{i \vartheta}$, where $\vartheta \in[0, \pi / 2]$. Suppose $A_{1}=[-c, 0], A_{2}=[-k c, k c]$ and $A_{3}=$ $[-k c / 2, c]$ and $A_{4}=A_{1}$, where $c>0$ and $k \in[0,1)$. Define $f, g: \cup_{i=1}^{3} A_{i} \rightarrow \cup_{i=1}^{3} A_{i}$ by

$$
\begin{aligned}
& f x= \begin{cases}-\frac{\alpha x}{c}, & \text { if } x>0, \\
0, & \text { otherwise, }\end{cases} \\
& g x=\frac{\beta x}{c},
\end{aligned}
$$

where $\alpha, \beta \geq 1$ with $\alpha<2 \beta \leq c$. Note that $f\left(A_{1}\right)=\{0\} \subseteq$ $[-k \beta, k \beta]=g\left(A_{2}\right), f\left(A_{2}\right)=[-k \alpha, 0] \subseteq[-k \beta / 2, \beta]=g\left(A_{3}\right)$, and $f\left(A_{3}\right)=[-\alpha, 0] \subseteq[-\beta, 0]=g\left(A_{1}\right)$. Hence $Y=A_{1} \cup A_{2} \cup$ $A_{3}$ is a cocyclic representation with respect to pair $(f, g)$ and the collection $\left\{A_{1}, A_{2}, A_{3}\right\}$. Define $\psi, \varphi: \mathbb{C}^{+} \rightarrow \mathbb{C}^{+}$by

$$
\begin{aligned}
& \psi(z)=\beta z, \\
& \varphi(z)= \begin{cases}\frac{\beta z}{4}, & \text { if } z \in\left[\theta, 2 \beta e^{i \vartheta}\right] \\
\beta z, & \text { if } t>2 \beta e^{i \vartheta} .\end{cases}
\end{aligned}
$$

Clearly $\psi$ is continuous and nondecreasing and $\varphi$ is lower semicontinuous with $\psi(z)=\varphi(z)=\theta$ if and only if $z=\theta$.

We show that condition (b) is satisfied:

(I) Let $x \in A_{1}$ and $y \in A_{2}$. If $y \in[-k c, 0]$, then $d(f x, f y)=\theta$ and hence (b) holds. If $y \in(0, k c]$, then we have

$$
\begin{aligned}
\psi(d(f x, f y)) & =\psi\left(d\left(0,-\frac{\alpha y}{c}\right)\right)=\frac{\alpha \beta}{c} y e^{i \vartheta} \\
& \preceq \frac{3 \beta^{2}}{4 c}(y-x) e^{i \vartheta}=\frac{3 \beta}{4} d\left(\frac{\beta x}{c}, \frac{\beta y}{c}\right) \\
& =\frac{3 \beta}{4} d(g x, g y) \preceq \frac{3 \beta}{4} U_{f, g}(x, y) \\
& =\beta U_{f, g}(x, y)-\frac{\beta}{4} U_{f, g}(x, y) \\
& =\psi\left(U_{f, g}(x, y)\right)-\varphi\left(U_{f, g}(x, y)\right) .
\end{aligned}
$$

(II) Let $x \in A_{2}, y \in A_{3}$. If $x \in[-k c, 0]$ and $y \in[-k c / 2,0]$, then $d(f x, f y)=\theta$ and hence (b) is satisfied. If $x \in$ $[-k c, 0]$ and $y \in(0, c]$, then we obtain that

$$
\begin{aligned}
\psi(d(f x, f y)) & =\psi\left(d\left(0,-\frac{\beta y}{c}\right)\right)=\frac{\beta^{2}}{c} y e^{i \vartheta} \\
& \prec \frac{3 \beta(\alpha+\beta)}{4 c} y e^{i \vartheta}=\frac{3 \beta}{4} d\left(-\frac{\alpha y}{c}, \frac{\beta y}{c}\right) \\
& =\frac{3 \beta}{4} d(f y, g y) \leq \frac{3 \beta}{4} U_{f, g}(x, y) \\
& =\beta U_{f, g}(x, y)-\frac{\beta}{4} U_{f, g}(x, y) \\
& =\psi\left(U_{f, g}(x, y)\right)-\varphi\left(U_{f, g}(x, y)\right) .
\end{aligned}
$$

When $x \in(0, k c]$ and $y \in[-k c / 2,0]$, we have

$$
\begin{aligned}
\psi(d(f x, f y)) & =\psi\left(d\left(-\frac{\alpha x}{c}, 0\right)\right)=\frac{\alpha \beta}{c} x e^{i \vartheta} \\
& \prec \frac{3 \beta(\alpha+\beta)}{4 c} x e^{i \vartheta}=\frac{3 \beta}{4} d\left(-\frac{\alpha x}{c}, \frac{\beta x}{c}\right) \\
& =\frac{3 \beta}{4} d(f x, g x) \preceq \frac{3 \beta}{4} U_{f, g}(x, y) \\
& =\beta U_{f, g}(x, y)-\frac{\beta}{4} U_{f, g}(x, y) \\
& =\psi\left(U_{f, g}(x, y)\right)-\varphi\left(U_{f, g}(x, y)\right) .
\end{aligned}
$$


For $x \in(0, k c]$ and $y \in(0, c]$, we obtain that

$$
\begin{aligned}
\psi(d(f x, f y)) & =\psi\left(d\left(-\frac{\alpha x}{c},-\frac{\alpha y}{c}\right)\right) \\
& =\frac{\alpha \beta}{c}|x-y| e^{i \vartheta} \prec \frac{3 \beta^{2}}{4 c}|x-y| e^{i \vartheta} \\
& =\frac{3 \beta}{4} d\left(\frac{\beta x}{c}, \frac{\beta y}{c}\right)=\frac{3 \beta}{4} d(g x, g y) \\
& \preceq \frac{3 \beta}{4} U_{f, g}(x, y) \\
& =\beta U_{f, g}(x, y)-\frac{\beta}{4} U_{f, g}(x, y) \\
& =\psi\left(U_{f, g}(x, y)\right)-\varphi\left(U_{f, g}(x, y)\right) .
\end{aligned}
$$

(III) Let $x \in A_{3}, y \in A_{1}$. If $x \in[-k c / 2,0]$, then $d(f x, f y)=\theta$ and (b) is satisfied. If $x \in(0, c]$, then we have

$$
\begin{aligned}
\psi(d(f x, f y)) & =\psi\left(d\left(-\frac{\alpha x}{c}, 0\right)\right)=\frac{\alpha \beta}{c} x e^{i \vartheta} \\
& \prec \frac{3 \beta(\alpha+\beta)}{4 c} x e^{i \vartheta}=\frac{3 \beta}{4} d\left(-\frac{\alpha x}{c}, \frac{\beta x}{c}\right) \\
& =\frac{3 \beta}{4} d(f x, g x) \preceq \frac{3 \beta}{4} U_{f, g}(x, y) \\
& =\beta U_{f, g}(x, y)-\frac{\beta}{4} U_{f, g}(x, y) \\
& =\psi\left(U_{f, g}(x, y)\right)-\varphi\left(U_{f, g}(x, y)\right) .
\end{aligned}
$$

Hence $f$ and $g$ satisfy all the conditions of Theorem 12 . Moreover, 0 is a unique common fixed point of $f$ and $g$ in $\cap_{i=1}^{3} A_{i}$.

We denote $\Upsilon=\left\{\phi: \mathbb{C}^{+} \rightarrow \mathbb{C}^{+}: \phi\right.$ is Lebesgue integrable with finite integral on each compact subset of $\mathbb{C}^{+}$ and, for each $\left.\theta \prec \varepsilon \in \mathbb{C}^{+}, \int_{\theta}^{\varepsilon} \varphi(z) d z>\theta\right\}$. As a consequence of Theorem 12, we obtain the following common fixed point result for mappings satisfying cocyclic contractive condition of integral type in a complex valued metric space.

Corollary 15. Let $(X, d)$ be a complex valued metric space, let $A_{1}, A_{2}, \ldots, A_{p}$, be nonempty closed subsets of $X$, and let $Y=$ $\cup_{i=1}^{p} A_{i}$. Suppose that $f, g: Y \rightarrow Y$ are mappings such that

(a) $Y$ has a cocyclic representation with respect to pair $(f, g)$ and to the collection $\left\{A_{i}: i=1,2, \ldots, p\right\}$;

(b) there exist control functions $\psi$ and $\varphi$ such that, for any $(x, y) \in A_{i} \times A_{i+1}, i=1,2, \ldots, p$,

$$
\begin{aligned}
\int_{\theta}^{\psi(d(f x, f y))} \phi(w) d w \preceq & \int_{\theta}^{\psi\left(U_{f, g}(x, y)\right)} \phi(w) d w \\
& -\int_{\theta}^{\varphi\left(U_{f, g}(x, y)\right)} \phi(w) d w
\end{aligned}
$$

is satisfied, where $\phi \in \Upsilon$ and

$$
\begin{aligned}
& U_{f, g}(x, y) \in\left\{d(g x, g y), \frac{d(f x, g x) d(f y, g y)}{1+d(g x, g y)},\right. \\
& \left.\frac{d(f x, g y) d(f y, g y)}{1+d(g x, g y)^{2}}, \frac{d(f x, g y) d(f y, g x)}{1+d(g x, g y)}\right\} \\
& \text { with } A_{p+1}=A_{1} .
\end{aligned}
$$

If $g\left(A_{i}\right)$ is a complete subspace of $X$ for each $i \in\{1,2, \ldots, p\}$, then $f$ and $g$ have a unique coincidence point $z \in \cap_{i=1}^{p} g\left(A_{i}\right)=$ $K$ provided that $f(K) \subseteq g(K) \subseteq K$ and $g(K)$ is closed. Moreover, if $f$ and $g$ are weakly compatible, then $f$ and $g$ have a unique common fixed point.

Proof. Define $\Psi: \mathbb{C}^{+} \rightarrow \mathbb{C}^{+}$by $\Psi(z)=\int_{\theta}^{z} \phi(w) d w$. From (69), it follows that

$$
\begin{aligned}
\Psi(\psi(d(f x, f y))) \preceq & \Psi\left(\psi\left(U_{f, g}(x, y)\right)\right) \\
& -\Psi\left(\varphi\left(U_{f, g}(x, y)\right)\right),
\end{aligned}
$$

which can be written as

$$
\psi^{*}(d(f x, f y)) \preceq \psi^{*}\left(U_{f, g}(x, y)\right)-\varphi^{*}(U(x, y)),
$$

where $\psi^{*}=\Psi \circ \psi$ and $\varphi^{*}=\Psi \circ \varphi$. Clearly, $\psi^{*}, \varphi^{*}$ : $\mathbb{C}^{+} \rightarrow \mathbb{C}^{+}, \psi^{*}$ is continuous and nondecreasing, $\varphi^{*}$ is lower semicontinuous, and $\psi^{*}(z)=\varphi^{*}(z)=\theta$ if and only if $z=\theta$. Hence, by Theorem 12, $f$ and $g$ have a unique common fixed point.

Remark 16. It is worth mentioning that the results obtained in this paper are distinct from the results in [8] in the following sense:

(1) The results in this paper are proved in the setup of complex valued metric spaces in which the distance between any two elements is a complex number but the results proved in [8] are in the setup of multiplicative metric spaces, where the distance between any two elements is a real number greater than or equal to 1 .

(2) The contractive conditions imposed on the mappings established in this paper are substantially different from contractions used in [8].

\section{Conflict of Interests}

The authors declare that there is no conflict of interests regarding the publication of this paper.

\section{Acknowledgments}

The second author thanks the Spanish Ministry of Economy and Competitiveness for its partial support of this work through Grant DPI2012-30651. The authors also thank the Basque Government for its support through Grant IT378-10 and the University of Basque Country for its support through Grant UFI 11/07. 


\section{References}

[1] Y. I. Alber and S. Guerre-Delabriere, "Principle of weakly contractive maps in Hilbert spaces," in New Results in Operator Theory and Its Applications, I. Gohberg and Y. Lyubich, Eds., vol. 98 of Operator Theory: Advances and Applications, pp. 7-22, Birkhäuser, Basel, Switzerland, 1997.

[2] I. Beg and M. Abbas, "Coincidence point and invariant approximation for mappings satisfying generalized weak contractive condition," Fixed Point Theory and Applications, vol. 2006, Article ID 74503, 7 pages, 2006.

[3] B. E. Rhoades, "Some theorems on weakly contractive maps," Nonlinear Analysis: Theory, Methods and Applications, vol. 47, no. 4, pp. 2683-2693, 2001.

[4] W. A. Kirk, P. S. Srinivasan, and P. Veeramini, "Fixed points for mappings satisfying cyclical contractive conditions," Fixed Point Theory, vol. 4, no. 1, pp. 79-89, 2003.

[5] M. Păcurar and I. A. Rus, "Fixed point theory for cyclic $\varphi$ contractions," Nonlinear Analysis, Theory, Methods and Applications, vol. 72, no. 3-4, pp. 1181-1187, 2010.

[6] E. Karapinar, "Fixed point theory for cyclic weak $\emptyset$-contraction," Applied Mathematics Letters, vol. 24, no. 6, pp. 822-825, 2011.

[7] M. Derafshpour and S. Rezapour, "On the existence of best proximity points of cyclic contractions," Advances in Dynamical Systems and Applications, vol. 6, no. 1, pp. 33-40, 2011.

[8] M. Abbas, M. De la Sen, and T. Nazir, "Common fixed points of generalized rational type cocyclic mappings in multiplicative metric spaces," Discrete Dynamics in Nature and Society, vol. 2015, Article ID 532725, 10 pages, 2015.

[9] M. Abbas, T. Nazir, and S. Romaguera, "Fixed point results for generalized cyclic contraction mappings in partial metric spaces," Revista de la Real Academia de Ciencias Exactas, Fisicas y Naturales A: Matematicas, vol. 106, no. 2, pp. 287-297, 2012

[10] R. P. Agarwal, M. A. Alghamdi, and N. Shahzad, "Fixed point theory for cyclic generalized contractions in partial metric spaces," Fixed Point Theory and Applications, vol. 2012, article 40, 2012.

[11] H. Aydi, C. Vetro, W. Sintunavarat, and P. Kumam, "Coincidence and fixed points for contractions and cyclical contractions in partial metric spaces," Fixed Point Theory and Applications, vol. 2012, article 124, 2012.

[12] P. Chaipunya, Y. J. Cho, W. Sintunavarat, and P. Kumam, "Fixed point and common fixed point theorems for cyclic quasicontractions in metric and ultrametric spaces," Advances in Pure Mathematics, vol. 2, no. 6, pp. 401-407, 2012.

[13] H. K. Nashine, W. Sintunavarat, and P. Kumam, "Cyclic generalized contractions and fixed point results with applications to an integral equation," Fixed Point Theory and Applications, vol. 2012, article 217, 2012.

[14] B. Piątek, "On cyclic Meir-Keeler contractions in metric spaces," Nonlinear Analysis, Theory, Methods \& Applications, vol. 74, no. 1, pp. 35-40, 2011.

[15] I. A. Rus, "Cyclic representation and fixed points," Annals of the Tiberiu Popoviciu Seminar of Functional Equations, Approximation and Convexity, vol. 3, pp. 171-178, 2005.

[16] W. Sintunavarat and P. Kumam, "Common fixed point theorem for cyclic generalized multi-valued contraction mappings," Applied Mathematics Letters, vol. 25, no. 11, pp. 1849-1855, 2012.

[17] O. Yamaod and W. Sintunavarat, "Some fixed point results for generalized contraction mappings with cyclic $(\alpha, \beta)$-admissible mapping in multiplicative metric spaces," Journal of Inequalities and Applications, vol. 2014, no. 1, article 488, 15 pages, 2014.
[18] A. Azam, B. Fisher, and M. Khan, "Common fixed point theorems in complex valued metric spaces," Numerical Functional Analysis and Optimization, vol. 32, no. 3, pp. 243-253, 2011.

[19] J. Ahmad, A. Azam, and S. Saejung, "Common fixed point results for contractive mappings in complex valued metric spaces," Fixed Point Theory and Applications, vol. 2014, article 67, 2014.

[20] S. A. Al-Mezel, H. H. Alsulami, E. Karapinar, and F. Khojasteh, "A note on fixed point results in complex-valued metric spaces," Journal of Inequalities and Applications, vol. 2015, article 3, 2015.

[21] B. S. Choudhury, N. Metiya, and P. Konar, "Fixed point results in partially ordered complex valued metric spaces for rational type expressions," Bangmod International Journal of Mathematical Computational Science, vol. 1, no. 1, pp. 55-62, 2015.

[22] B. S. Choudhury, N. Metiya, and P. Konar, "Fixed point results for rational type contraction in partially ordered complex valued metric spaces," Bulletin of the International Mathematical Virtual Institute, vol. 5, no. 1, pp. 73-80, 2015.

[23] M. Öztürk, "Common fixed point theorems satisfying contractive type conditions in complex valued metric spaces," Abstract and Applied Analysis, vol. 2014, Article ID 598465, 7 pages, 2014.

[24] R. K. Verma and H. K. Pathak, "Common fixed point theorems using property (E.A) in complex-valued metric spaces," Thai Journal of Mathematics, vol. 11, no. 2, pp. 347-355, 2013.

[25] M. Abbas, B. Fisher, and T. Nazir, "Well-posedness and periodic point property of mappings satisfying a rational inequality in an ordered complex valued metric space," Scientific Studies and Research Series: Mathematics and Informatics, vol. 22, no. 1, pp. 5-24, 2012.

[26] H. K. Nashine, M. Imdad, and M. Hasan, "Common fixed point theorems under rational contractions in complex valued metric spaces," The Journal of Nonlinear Science and Applications, vol. 7, pp. 42-50, 2014.

[27] M. Abbas and G. Jungck, "Common fixed point results for noncommuting mappings without continuity in cone metric spaces," Journal of Mathematical Analysis and Applications, vol. 341, no. 1, pp. 416-420, 2008.

[28] R. H. Haghi, S. Rezapour, and N. Shahzad, "Some fixed point generalizations are not real generalizations," Nonlinear Analysis, Theory, Methods and Applications, vol. 74, no. 5, pp. 1799-1803, 2011. 


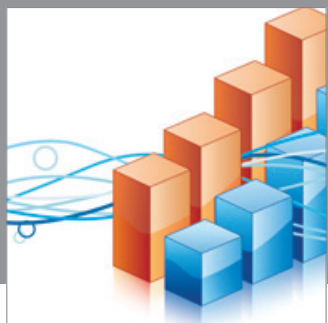

Advances in

Operations Research

mansans

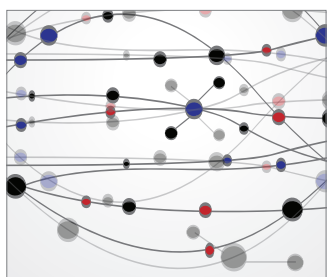

The Scientific World Journal
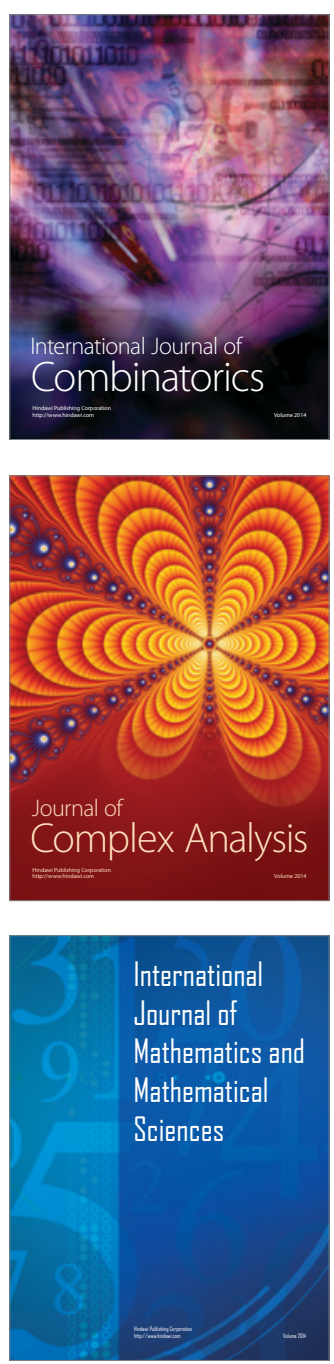
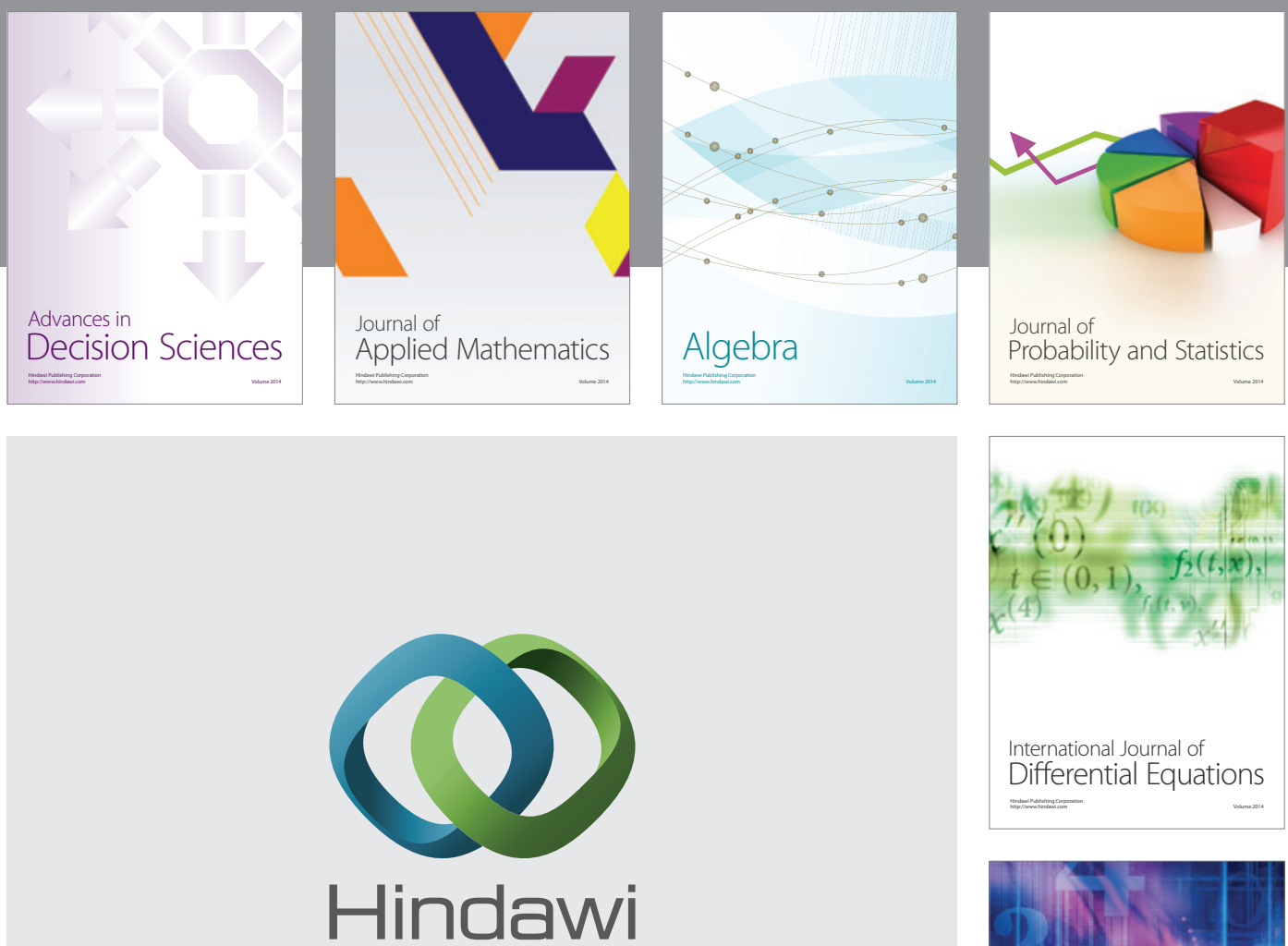

Submit your manuscripts at http://www.hindawi.com
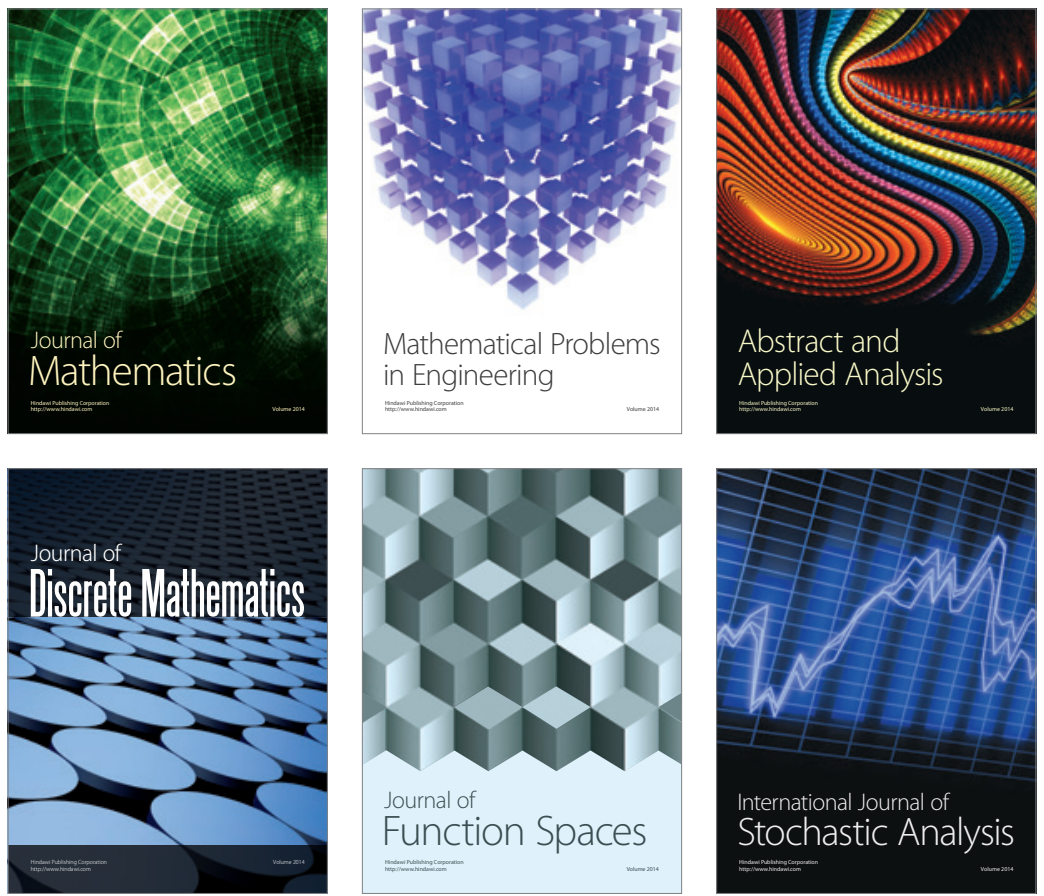

Journal of

Function Spaces

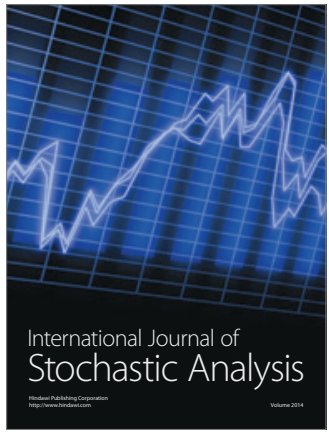

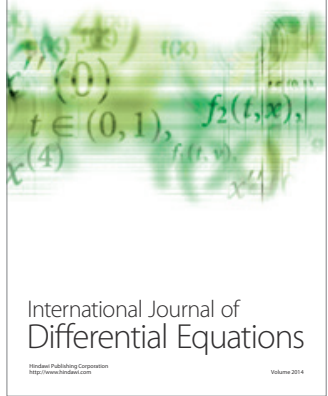
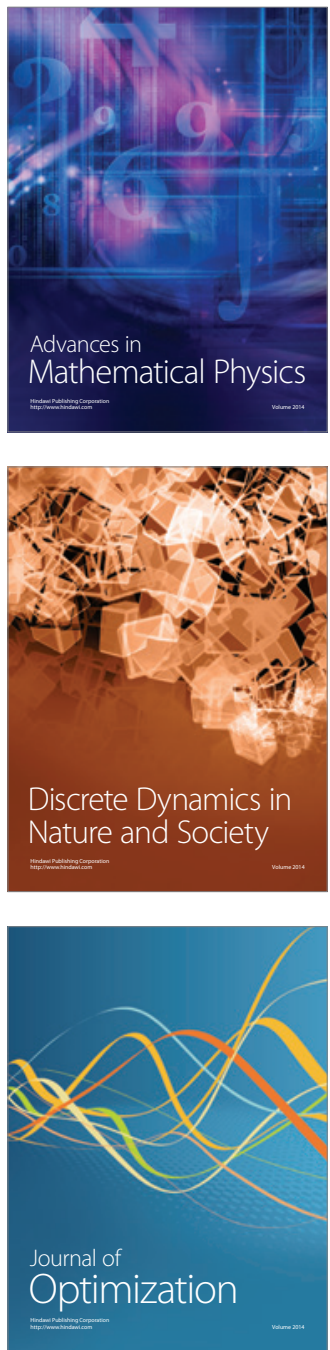